\title{
Monte-Carlo-Methode zur Verringerung von Messfehlern bei der Materialparameterbestimmung mit Hohlraumresonatoren
}

\author{
Monte Carlo method for the reduction of measurement errors in the material parameter estimation \\ with cavities
}

DOI 10.1515/teme-2020-0027

Zusammenfassung: Die quantitative Bestimmung von Materialparameterverläufen in Hohlraumresonatoren ist eine relativ neue Methode zur echtzeitfähigen Überwachung verfahrenstechnischer Prozesse. Dazu werden elektromagnetische Resonanzen des Hohlraumresonators als Eingangsdaten für die Rückrechnung (Inversion) verwendet. Der Rückrechenalgorithmus ist allerdings empfindlich auf Störungen (= Messabweichungen) der Eingangsdaten und neigt zum Divergieren. In dieser Arbeit wird ein auf der Monte-Carlo-Methode beruhender Korrekturalgorithmus präsentiert, der für ein konvergentes Verhalten des Rückrechenalgorithmus sorgt.

Schlüsselwörter: Materialparameter, Materialparameterverteilung, Hohlraumresonator, Messfehler.

\begin{abstract}
The quantitative determination of material parameterdistributions in resonant cavities is a relatively new method for the real-time monitoring of chemical processes. For this purpose, electromagnetic resonances of the cavity resonator are used as input data for the reverse calculation (inversion). However, the reverse calculation algorithm is sensitive to disturbances (= measurement errors) of the input data and tends to diverge. In this work a correction algorithm based on the Monte Carlo method is presented which ensures a convergent behavior of the reverse calculation algorithm.
\end{abstract}

Keywords: material parameter, material parameter distribution, cavity resonator, measurement error.

\footnotetext{
*Korrespondenzautor: Ronny Peter, Lehrstuhl für Mess- und Regeltechnik, Universität Bayreuth, Universitätsstraße 30, 95447 Bayreuth, E-Mail: ronny.peter@uni-bayreuth.de

Gerhard Fischerauer, Lehrstuhl für Mess- und Regeltechnik, Universität Bayreuth, Universitätsstraße 30, 95447 Bayreuth, E-Mail mrt@uni-bayreuth.de
}

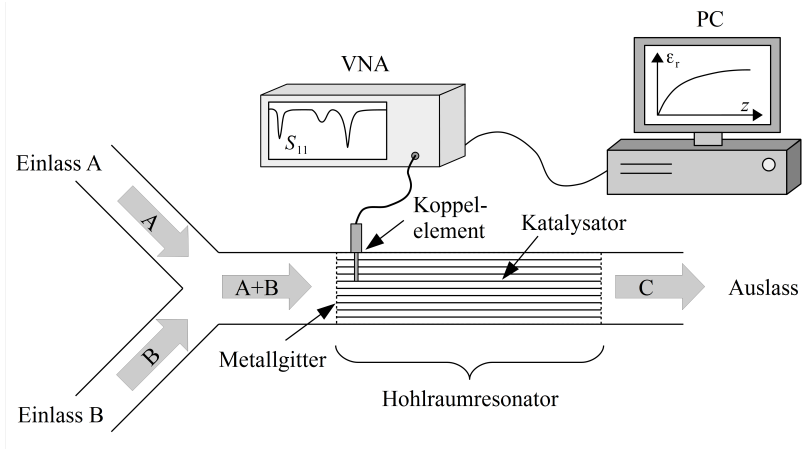

Abb. 1: Schematischer Aufbau einer Prozessüberwachung mit Hohlraumresonatoren durch Bestimmung des Materialparameterverlaufs im Resonator.

\section{Einleitung}

Die Methode der Hohlraumresonatorstörung ist seit Jahren bekannt und wird als Labormessmethode zur Bestimmung der elektrischen Materialparameter (Permittivität und Leitfähigkeit) präparierter homogener Proben verwendet [1]. Eine andere interessante Anwendung, zu der gegenwärtig geforscht wird, ist die Messung von Materialparameterverläufen in-situ, also im laufenden Prozess. Zum Beispiel lässt sich ein Rohrreaktor durch metallische Gitter am Ein- und Auslass zum elektrisch abgeschirmten Hohlraumresonator umwandeln, gleichzeitig können Fluide praktisch ungehindert durch den Reaktor strömen (Abb. 1).

Der Resonator wird durch ein Koppelelement mit einem Netzwerkanalysator verbunden, sodass Streuparameterspektren, etwa der Reflexionsfaktor $\underline{S}_{11}(f)$, gemessen werden können. Durch sorgfältiges De-Embedding können unbelastete Resonanzparameter (Resonanzfrequenz $f_{0}$ und unbelastete Güte $Q_{0}$ ) aus dem Spektrum extrahiert 
werden [4]. Anhand der unbelasteten Parameter mehrerer Resonanzen kann auf die Materialparameterverteilung im Resonator rückgerechnet werden [5]. Dabei wird zunächst unterstellt, dass die Materialparameter nur in longitudinaler Richtung (im Beispiel: in Fließrichtung der Reagenzien) variieren, in transversaler Richtung aber als homogen genähert werden können. Dies ist der Sollzustand der meisten Prozesse (z. B. beim Drei-Wege-Abgaskatalysator [7]), weil transversale Inhomogenitäten die Prozesseffizienz beeinträchtigen.

\section{Problem der Materialparameterbestimmung}

\subsection{Vorwärtsmodellierung}

Der Resonator wird im mathematischen Modell in Segmente mit konstanten Materialparametern diskretisiert (Abb. 2). Ziel ist es, anhand gemessener Resonanzparameter den Segmenten die Materialparameter zuzuordnen. Dazu existieren zwei Verfahren, eines mit [6] und eines ohne Nebenbedingung [5].

\subsection{Inversion}

Beide erwähnten Inversionsverfahren leiden unter Instabilitäten. Die Rückrechenalgorithmen konvergieren regelmäßig nur dann, wenn a) die Eingangsparameter (sprich: die gemessenen Resonanzparameter) nur sehr wenig von ihren wahren Werten abweichen (rel. Messabweichung $\tilde{\varepsilon}_{f} \ll 1 \%$ ) und wenn b) Startwerte nahe der Lösung vorgegeben werden (Abweichung ca. $\pm 10-20 \%$ ). In der Realität ist Bedingung b) nicht zwangsläufig gegeben,

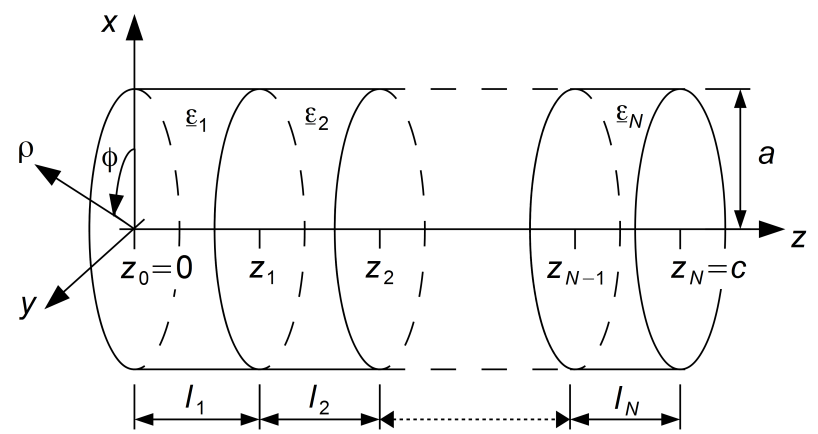

Abb. 2: Mathematische Modellvorstellung eines aus $N$ homogenen Segmenten bestehenden inhomogenen zylindrischen Hohlraumresonators. kann aber mit überschaubarem Aufwand erreicht werden (z. B. Multi-Start-Algorithmus). Problematisch ist hingegen Bedingung a). Derart präzise Messungen sind in der Realität oft sehr schwer oder gar nicht erreichbar. Es zeigte sich, dass der Rückrechenalgorithmus insbesondere in denjenigen Fällen Konvergenzprobleme aufwies, in denen der Messfehler einer einzelnen Resonanz deutlich größer war als der Messfehler der übrigen Resonanzen.

Messabweichungen entstehen bei der Resonanzparameterbestimmung auf unterschiedlichste Arten. Zu nennen wären z. B. Geometrieabweichung des Resonators, zu starke Materialparameteränderung innerhalb der als konstant genäherten Segmente, Messabweichungen bei der Spektrenmessung, Fehler im Modell zur Resonanzparameterextraktion, Fehler der Modellfunktion (z. B. wegen Rauschens der Messwerte), De-Embedding-Fehler.

\section{Inversionsalgorithmus auf Basis der Monte-Carlo-Methode}

\subsection{Prinzip}

Wir stellen als neuartiges Verfahren die Monte-CarloMethode [3] vor, um einen „Kompensationsfehler“ zu den gemessenen fehlerbehafteten Resonanzparametern zu addieren. Die normalverteilten zufälligen Kompensationsfehler können den Rückrechenalgorithmus auf zwei Arten beeinflussen:

1. Algorithmus konvergiert (trotzdem) nicht;

2. Algorithmus konvergiert zu einer Lösung $L_{1}^{*} \in L_{2}$.

Dabei bezeichnen $L_{1}$ die Lösung bei fehlerlosen Eingangsdaten, $L_{1}^{*}$ eine einzelne Lösung bei fehlerbehafteten Eingangsdaten und zufälligem Kompensationsfehler und $L_{2}$ die Menge aller $L_{1}^{*}$.

Das Ziel ist eine ausreichende Verringerung der Messfehler, sodass der Algorithmus konvergiert und idealerweise ein globales Optimum findet. Durch mehrmalige Wiederholung der Rückrechnung mit unterschiedlichem Fehler entsteht eine normalverteilte Lösungsmenge $L_{2}$. Deren Durchschnitt ist eine Schätzung von $L_{1}$.

\subsection{Validierung}

In einem Validierungsbeispiel 1 wurden vier Resonanzfrequenzen des rechteckigen Hohlraumresonators gemäß Abb. 3 vorwärts gerechnet. Zu diesen wurde ein normalverteilter Zufallsfehler $(< \pm 1 \%)$ hinzuaddiert. Diese fehlerbe- 


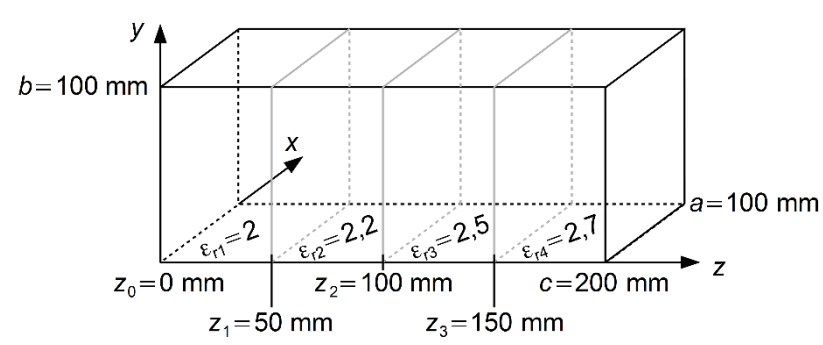

Abb. 3: Mathematisches Modell eines durch 4 Segmente konstanter Permittivität genäherten rechteckigen Hohlraumresonators.

hafteten Resonanzfrequenzen dienten als fehlerbehaftete synthetische Messwerte.

Für die Rückrechnung wurden verschiedene normalverteilte zufällige Kompensationsfehler addiert. Dies wurde etwa 135000 mal mit jeweils neuen Kompensationsfehlern wiederholt. Am Ende standen 2500 Lösungen, die aus einer konvergierenden Rückrechnung stammten und zusätzlich das Kriterium der physikalischen Plausibilität erfüllten. Außerdem war bei diesen Lösungen das Residuum $r$ kleiner als eine vorgegebene Grenze, sodass von einem globalen Optimum ausgegangen werden kann. Das Residuum ist dabei definiert durch

$$
r=\sum_{m n p}\left(f_{m n p}^{\mathrm{TE}}\left(\hat{\vec{\varepsilon}}_{r}\right)-\hat{f}_{m n p}^{\mathrm{TE}}\left(\vec{\varepsilon}_{r}\right)\right)^{2} .
$$

Dabei bezeichnen $\hat{f}_{m n p}^{\mathrm{TE}}\left(\vec{\varepsilon}_{r}\right)$ die aus der (synthetischen) Spektrenmessung extrahierte Resonanzfrequenz des TE $m n p$-Modus und $f_{m n p}^{\mathrm{TE}}\left(\hat{\vec{\varepsilon}}_{r}\right)$ die auf Basis der geschätzten Materialparameter vorwärts gerechnete Resonanzfrequenz desselben Modus.

Die Lösungsmenge bei diesem Beispiel ist in Abb. 4 als Histogramm dargestellt. Die durchschnittlichen rückgerechneten Permittivitäten der Segmente weichen betragsmäßig um weniger als $5 \%$ von den vorgegebenen Permittivitäten ab.

Im Validierungsbeispiel 2 entstammen die Resonanzparameter der Simulation mit dem kommerziellen Finite-Elemente-Programm Ansys HFSS. Es wurde zunächst das $\underline{S}_{11}$-Spektrum eines mit sechs verschiedenen Materialien gefüllten stiftgekoppelten Zylinderresonators (vgl. Abb. 2) simuliert (1-5 GHz, 8001 Frequenzpunkte), aus dem dann die Resonanzparameter extrahiert wurden. Zur Extraktion wurde Gleichung (9) aus [2, S. 375] verwendet. Um den "Messfehler" künstlich zu erhöhen, wurde der Resonator in einem geradlinigen Koordinatensystem anstatt in einem, für die Zylindergeometrie besser geeigneten, krummlinigen Koordinatensystem, vernetzt. Außerdem wurde kein De-Embedding durchgeführt. Dieses Vorgehen sorgt für künstliche Messfehler, bietet aber im

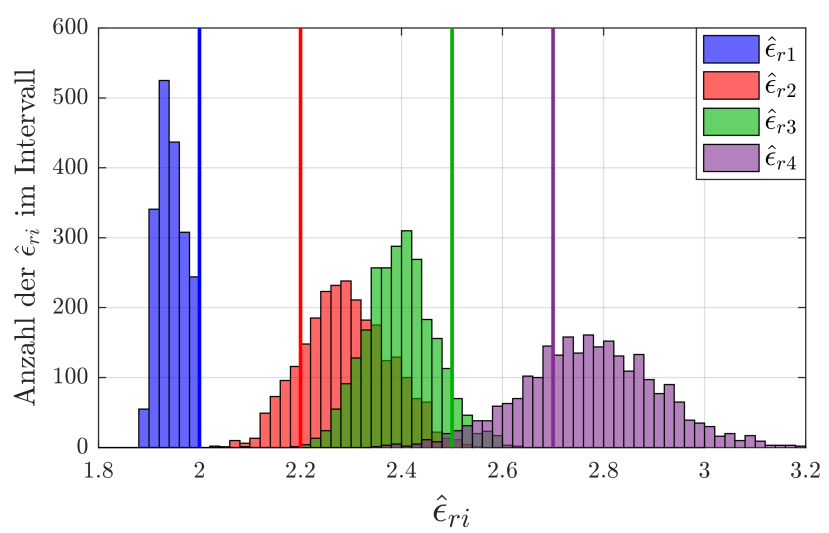

Abb. 4: Ergebnisse der Materialparameterschätzung auf der Grundlage fehlerbehafteter Resonanzfrequenzen (synthetischer Messwerte) mit der Monte-Carlo-Methode. Senkrechte Linien: vorgegebene Permittivitäten.

Vergleich zu einem realen Experiment den Vorteil, dass die Materialparameterverteilung exakt bekannt ist. Im Gegensatz zu Beispiel 1 enthalten die Resonanzfrequenzen in Beispiel 2 mindestens einen systematischen Fehleranteil, da das Volumen des simulierten Resonators (abgeschrägter Zylinder) geringer ist als das mathematische Modell (perfekter Zylinder). Zusätzliche Fehleranteile durch numerisches Rauschen könnten normalverteilt sein.

Das Simulationsmodell des Resonators besteht aus sechs zylindrischen Segmenten unterschiedlicher Permittivität mit jeweils $25 \mathrm{~mm}$ Länge und $50 \mathrm{~mm}$ Radius. Zur numerischen Berechnung wurden diese in mehrere tausend Tetraeder zerlegt. Bei der Rückrechnung wurde ein mathematisches Modell mit ebenfalls sechs Segmenten verwendet. Folglich würde, bei idealer Bestimmung der unbelasteten Resonanzparameter, die Rückrechnung die vorgegebenen Materialparameter in allen Segmenten exakt (von vernachlässigbaren numerischen Fehlern abgesehen) wiedergeben.

Die künstlich herbeigeführte Fehlbestimmung der Resonanzparameter sorgt bei der Rückrechnung zum einen für eine Abweichung von den vorgegebenen Werten, zum anderen aber auch für einen divergenten Rückrechenalgorithmus. Während ersteres in vielen Anwendungsfällen noch akzeptabel wäre, ist die Divergenz eines Algorithmus ein Ausschlusskriterium der Methode.

Für Beispiel 2 wurden sechs Resonanzfrequenzen aus den simulierten Spektren extrahiert. Zur Veranschaulichung ist in Abb. 5 exemplarisch der Betrag der elektrischen Feldstärke eines Modus dargestellt. Gut zu erkennen ist der Einfluss der unterschiedlichen Permittivitäten auf die Feldverteilung/-intensität des Modus. 


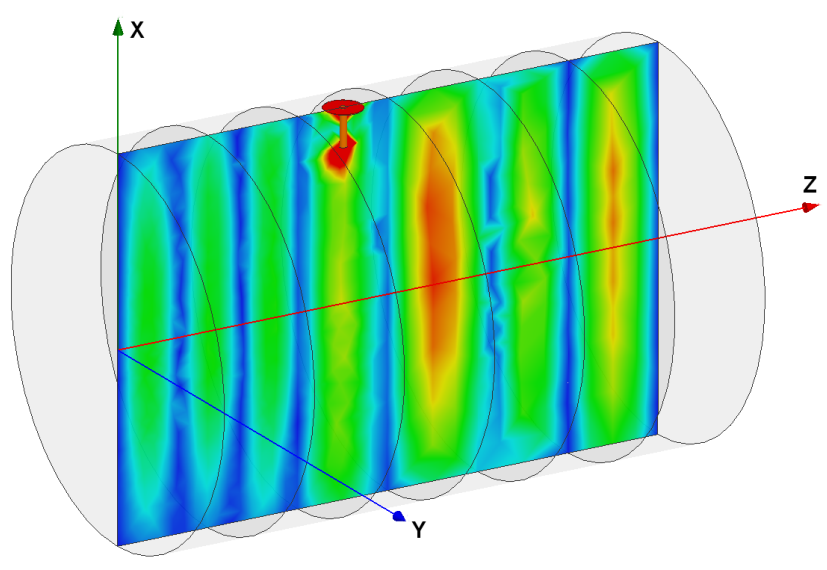

Abb. 5: Betrag der elektrischen Feldstärke des $\mathrm{TE}_{117}$-Modus im Resonator in der Schnittebene $y=0$.

Tab. 1: Übersicht der Iterations- und Lösungsanzahlen für verschiedene Skalierungsfaktoren.

\begin{tabular}{lrrr}
\hline$\nu \cdot 10^{3}$ & 7,5 & 5,0 & 2,5 \\
\hline Inversionen (gesamt) & 14696 & 6292 & 3370 \\
Phys. sinnvolle Lösungen & 381 & 390 & 405 \\
Phys. sinnvolle Lösungen mit kleinem & 250 & 250 & 250 \\
Residuum & & & \\
\hline
\end{tabular}

Die Eingangsdaten wurden mit einem Kompensationsfehler gemäß

$$
\hat{f}_{m n p}^{\prime \mathrm{TE}}=\hat{f}_{m n p}^{\mathrm{TE}}(1+n \nu)
$$

beaufschlagt. Dabei ist $n$ eine nach $N(\mu=0, \sigma=1)$ normalverteilte Zufallsgröße, $\nu$ ein Skalierungsfaktor und $\hat{f}_{m n p}^{\prime \mathrm{TE}}$ die mit dem Kompensationsfehler beaufschlagte Resonanzfrequenz.

Dies wurde solange mit jeweils neuen Zufallsfehlern wiederholt, bis 250 physikalisch sinnvolle Lösungen mit kleinem Residuum berechnet waren. Dieses Vorgehen wurde für drei verschiedene Skalierungsfaktoren $\nu$ durchgeführt.

Die jeweils nötige Gesamtzahl der Inversionen und die der physikalisch sinnvollen Lösungen mit zu großem Residuum sind in Tab. 1 aufgelistet. Aufgrund der Größenordnung der Fehler in der Simulation scheint ein Skalierungsfaktor $\nu=0,0025$ am besten zur Rückrechnung geeignet, da dort die wenigsten Iterationen nötig waren. Bei einem größeren Fehler der gemessenen Resonanzfrequenzen wäre vermutlich ein größerer Skalierungsfaktor geeigneter; dies bleibt aber noch zu untersuchen.

Exemplarisch für die übrigen Skalierungsfaktoren ist ein Histogramm der 250 Lösungen bei $\nu=0,0025$ in Abb. 6 dargestellt. Das Histogramm zeigt erneut, in guter Näherung, normalverteilte Lösungen.

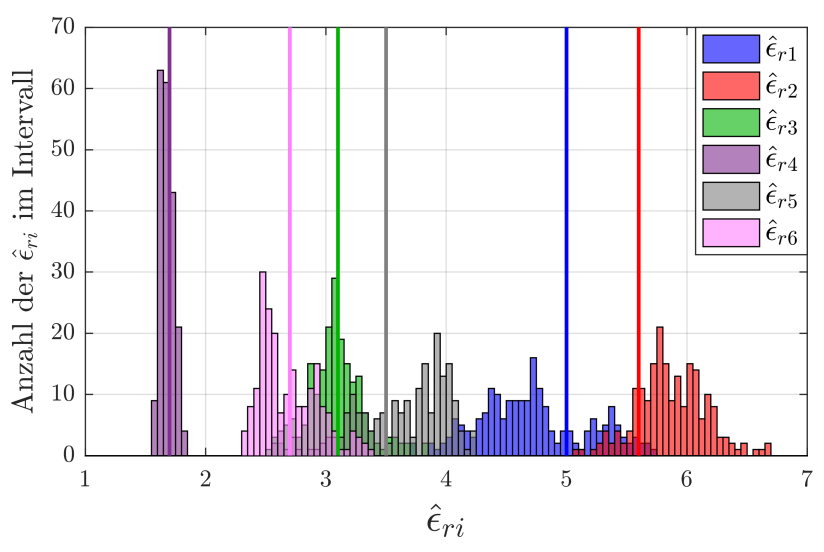

Abb. 6: Wie Abb. 4, aber für Beispiel $2(\nu=0,0025)$.

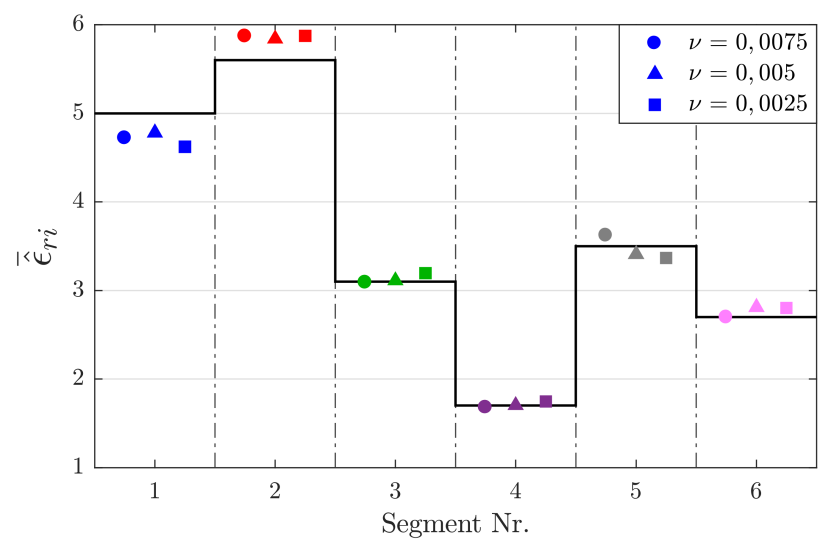

Abb. 7: Ergebnisse der Rückrechnung (Beispiel 2) mit der vorgestellten Monte-Carlo-Methode für verschiedene Werte des Skalierungsfaktors $\nu$. Dargestellt ist der Mittelwert aller physikalisch sinnvollen Lösungen mit Residuum kleiner $100 \mathrm{~Hz}^{2}$.

Die Fehler der durchschnittlichen Permittivitäten der einzelnen Segmente sind in Abb. 7 dargestellt. Es scheint, als ob der Skalierungsfaktor keinen Einfluss auf das Messergebnis hat. Dies würde man auch vermuten, da jeweils die gleiche Anzahl physikalisch sinnvoller Lösungen mit dem gleichen geringen Residuum verwendet wurde. Der Skalierungsfaktor bestimmt folglich nur die Wahrscheinlichkeit, mit der eine solche Lösung gefunden wird (vgl. Tab. 1).

Auch in Beispiel 2 weichen die Mittelwerte der geschätzten Permittivitäten nur geringfügig von den tatsächlichen Permittivitäten ab. In der Mehrzahl der Fälle betrug die Abweichung weniger als $3 \%$. Dabei muss bedacht werden, dass die vorgestellte Monte-Carlo-Methode nicht zur Verringerung des Fehlers bei der Bestimmung der Permittivitäten dient, sondern lediglich die Konvergenz des Rückrechenalgorithmus sicherstellen soll. Die fehlerbehafteten Eingangsdaten sind für den Fehler in der Bestimmung der Permittivitäten maßgeblich. 


\subsection{Bewertung}

Die Materialparameter, die der Monte-Carlo-gestützte Inversionsalgorithmus auf der Basis „gemessener“ (synthetisch mit Fehlern erzeugter) Resonanzparameter geschätzt hat, wichen in den Validierungsbeispielen um maximal $7,5 \%$ von den wahren Werten ab. Inwiefern dies für einen messtechnischen Einsatz der Methode akzeptabel ist, muss im Einzelfall geprüft werden. Es steht freilich nur die unattraktive Alternative zur Verfügung, auf die Kenntnis einer ortsabhängigen Materialparameterverteilung ganz zu verzichten, weil bisherige Inversionsalgorithmen (ohne MonteCarlo-Ansatz) divergieren. Bei elektrochemischen Systemen ist die Kenntnis ortsabhängiger (z. B. Katalysator-) Zustände im Feld bislang höchstens ein Wunschtraum, und selbst eine grobe Kenntnis stellt einen bemerkenswerten Fortschritt dar.

Der Rechenaufwand des Monte-Carlo-Ansatzes ist selbstverständlich höher als bei einer einzelnen Inversion. Allerdings lässt sich die Methode sehr gut parallelisieren. Bei massiver paralleler Berechnung, z. B. auf einer Grafikkarte, erhöht sich die Rechenzeit gegenüber einer einmaligen Inversion nur unerheblich.

\section{Zusammenfassung}

Die Methode der Hohlraumresonatorstörung zur Bestimmung von axial inhomogener Materialparameterverteilung ist vielseitig anwendbar und kann z. B. zur Echtzeitüberwachung verfahrenstechnischer Prozesse genutzt werden. Ein Problem, das in der Praxis auftritt, ist die regelmäßige Divergenz des Inversionsalgorithmus durch fehlerhafte Eingangsdaten (= gemessene Resonanzparameter). Das hier vorgestellte neue Inversionsverfahren nutzt die MonteCarlo-Methode, um die Eingangsdaten mit zufälligen normalverteilten „Kompensationsfehlern“ zu beaufschlagen. Die mehrfache Wiederholung dieses Vorgehens führt in der Regel zu mehreren konvergenten Inversionsergebnissen. Diese sind jeweils für sich genommen eine Schätzung der tatsächlichen Lösung bei fehlerfreien Eingangsdaten. Werden mehrere konvergente Lösungen zusammengenommen, entsteht eine noch bessere Schätzung.

Danksagung: Die vorgestellten Arbeiten wurden von der Deutschen Forschungsgemeinschaft (DFG) unter Projektnummer 389867475 gefördert.

\section{Literatur}

[1] L. F. Chen. Microwave electronics: Measurement and materials characterization. Wiley, 2004.

[2] S. A. Dyer, editor. Survey of instrumentation and measurement. John Wiley \& Sons, 2001.

[3] G. S. Fishman. Monte Carlo: Concepts, Algorithms, and Applications. Springer, 1995.

[4] R. Peter und G. Fischerauer. De-embedding method for strongly coupled cavities. IEEE Trans. Microw. Theory Techn., 66(4):2025-2033, Apr 2018. 10.1109/TMTT.2018.2791934.

[5] R. Peter und G. Fischerauer. Measurement of axially inhomogeneous permittivity distributions in resonant microwave cavities. IEEE Trans. Microw. Theory Tech., 67(6):2433-2442, Jun 2019. DOI: 10.1109/TMTT.2019.2910177.

[6] R. Peter und G. Fischerauer. In-situ measurement of permittivity distributions in reactors by cavity perturbation. Meas. Sci. Techn., 31(9), Jun 2020. 10.1088/1361-6501/ab6add.

[7] F. Sbrazzi, P. Faraldi und A. Soldati. Appraisal of threedimensional numerical simulation for sub-micron, particle deposition in a microporous ceracmiuc filter. Chem. Eng. Sci., 60(23):6551-6563, Dec 2005 